\title{
Spondyloepiphyseal dysplasia, Maroteaux type
}

INSERM

\section{Source}

INSERM. (1999). Orphanet: an online rare disease and orphan drug data base.

Spondyloepiphyseal dysplasia, Maroteaux type. ORPHA:263482

Spondyloepiphyseal dysplasia, Maroteaux type is a very rare type of spondyloepiphyseal dysplasia (see this term) described in fewer than 10 patients to date and characterized clinically by dysplastic epiphyses, short stature appearing in infancy, short neck, short and stubby hands and feet, scoliosis, genu valgum, abnormal pelvis, osteoporosis and osteoarthritis. 